\title{
A High Voltage Asymmetric Waveform Generator for FAIMS
}

\author{
Jesse D. Canterbury, ${ }^{\mathrm{a}}$ James Gladden, ${ }^{\mathrm{b}}$ Lon Buck, ${ }^{\mathrm{b}}$ Roy Olund, ${ }^{\mathrm{b}}$ and \\ Michael J. MacCoss ${ }^{a}$ \\ ${ }^{a}$ Department of Genome Sciences, University of Washington, Seattle, Washington, USA \\ ${ }^{\mathrm{b}}$ Department of Chemistry, University of Washington, Seattle, Washington, USA
}

\begin{abstract}
High field asymmetric waveform ion mobility spectrometry (FAIMS) has been used increasingly in recent years as an additional method of ion separation and selection before mass spectrometry. The FAIMS electrodes are relatively simple to design and fabricate for laboratories wishing to implement their own FAIMS designs. However, construction of the electronics apparatus needed to produce the required high magnitude asymmetric electric field oscillating at a frequency of several hundred kilohertz is not trivial. Here we present an entirely custom-built electronics setup capable of supplying the required waveforms and voltages. The apparatus is relatively simple and inexpensive to implement. We also present data acquired on this system demonstrating the use of FAIMS as a gas-phase ion filter interface to an ion trap mass spectrometer. (J Am Soc Mass Spectrom 2010, 21, 1118-1121) (c) 2010 American Society for Mass Spectrometry
\end{abstract}

$\mathrm{H}$ igh-field asymmetric waveform ion mobility spectrometry (FAIMS), also called field-asymmetric ion mobility spectrometry and, more generally, differential ion mobility spectrometry, has been used for decades $[1,2]$ as an analytical tool in a wide variety of applications, from fundamental studies of ion mobility to the comprehensive analyses of complex protein mixtures common in proteomics studies. In all of these studies, the technique is generally similar, though the details can vary widely (the recent book by Shvartsburg reviews the science and history of FAIMS [3]). Analyte ions are injected into a stream of neutral carrier gas molecules, which flows into a region enclosed between two electrodes. Ions are then subjected to a high magnitude, high-frequency asymmetric rf field in which the positive portion of the waveform is half as long (in time) and twice as large (in magnitude) as the negative portion, so that the total field sums to zero over one period. For an electric field of roughly $5 \mathrm{kV} / \mathrm{cm}$ or greater, ions can be separated on the basis of their differential mobility, $\left(\mathrm{K}_{\mathrm{h}}-\mathrm{K}\right) / \mathrm{K}$, where $\mathrm{K}_{\mathrm{h}}$ is the mobility during the high voltage portion of the waveform and $\mathrm{K}$ is the mobility during the low-voltage portion, as has been described in detail elsewhere.

Although simple in principle, the electronics apparatus necessary to generate the high magnitude asymmetric field required in FAIMS is challenging to implement for commonly used devices in which the analytical gap width between the two electrodes is $0.5-2.5 \mathrm{~mm}$ (recently developed FAIMS microchips place less stringent requirements on the waveform generator, though re-

Address reprint requests to Dr. M. J. MacCoss, Department of Genome Sciences, University of Washington, Box 355065, W. H. Foege Building S113, 1705 NE Pacific St., Seattle, WA 98195, USA. E-mail: maccoss@u. washington.edu quired frequencies may be as high as $30 \mathrm{MHz}[4,5])$. Indeed, most applications have relied on commercial electronics [6-8] that are either used as delivered or adapted (mostly in terms of connectivity) to different experimental situations. Details of the electronics apparatus necessary for FAIMS have appeared infrequently in the published literature since the early paper by Krylov examining several different designs [9]. A paper by Miller et al. briefly described their implementation of a generator using a flyback transformer design, which was capable of peak-to-peak voltages of $1400 \mathrm{~V}$ at $2 \mathrm{MHz}$, designed around a micro-machined set of electrodes [10]. More recently, work by Papanastasiou et al. describes a generator capable of $\sim 1200 \mathrm{~V}$ peak-topeak generation of square waves with variable duty cycles, using a fast switching scheme [11]. While innovative, these designs do not supply the $\sim 6 \mathrm{kV}$ peak-topeak required for the "macro"-machined FAIMS devices employing gap widths of $2 \mathrm{~mm}$ or more that have been used in the majority of FAIMS studies to date. A recent design capable of higher voltages appeared at a national meeting [12]. This design takes an innovative approach to coupling the rf signal to the FAIMS electrodes and employs an aggressive approach to cooling the generator; however, details of this design have not yet been published. Additionally, recent work in adding a ripple voltage to an extractor electrode in a FAIMS device used an asymmetric waveform generator capable of a maximum peak voltage of $3.6 \mathrm{kV}$, but details of the generator were not included in the paper [13].

Here we describe our FAIMS electronics setup and demonstrate its use in ion separations interfaced with an ion trap mass spectrometer. The electronics setup is relatively simple and inexpensive and produces the bi-sinusoidal waveform most commonly used in FAIMS 
at a peak-to-peak voltage of $6 \mathrm{kV}$ or more, and at a frequency of about $715 \mathrm{kHz}$. This apparatus can be adjusted to allow output of unusual FAIMS waveforms $[14,15]$, which may expand the scope and usefulness of the FAIMS technique. Our setup uses modular electronics (arbitrary waveform generator, rf power amplifier) commonly found in most laboratories as well as a customdesigned coupling network that is inexpensive to construct. All plans and schematics for our FAIMS apparatus are available in the Supplementary Material, which can be found in the electronic version of this article.

\section{Methods}

\section{Electronics Design}

Overview. A schematic of our experimental apparatus is shown in Figure 1. The bi-sinusoidal asymmetric waveform (see Supplementary Material for details) is generated by an arbitrary waveform generator (HP $33120 \mathrm{~A}$ ), which is connected to the input of a $100 \mathrm{~W} \mathrm{rf}$ power amplifier (ENI 2100L). The output of the power amplifier is connected to a doubly resonant coupling network consisting of series and parallel inductorcapacitor (LC) networks, which float on a small DC

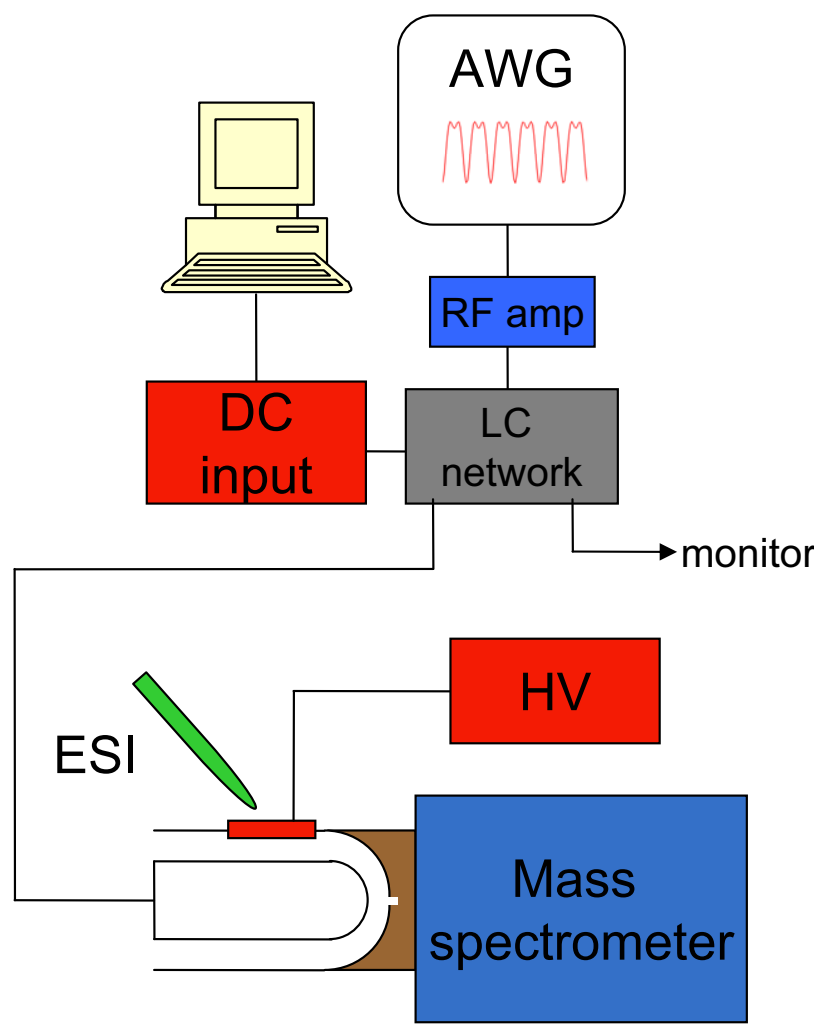

Figure 1. Block diagram of experimental setup. The asymmetric waveform is generated in the arbitrary waveform generator (AWG), amplified in the rf amp, and further amplified in the LC network, which is connected to the FAIMS electrodes. The CV is supplied by a DC input, controlled by a LabView program, and the waveform is monitored by oscilloscope. A curtain voltage is supplied by a high voltage supply. Carrier gas inlet is not shown. See Figure 2 for a schematic of the LC network. voltage. This DC voltage serves as the FAIMS compensation voltage (supplied by an American Reliance PD120-0.3DA power supply). The output of this network is connected directly to the inner electrode on the FAIMS device with a short section of RG-8 cable terminated with SHV connectors.

\section{Coupling Network}

The coupling network depicted in Figure 2 consists of a series $2 x L-C$ circuit followed by a parallel L-2xC circuit with the FAIMS electrodes and the high-voltage cable making up part of the parallel L-C capacitance. These 2 LC elements combine to produce a single network with two resonant frequencies. When the circuit values are chosen such that L-series equals twice the L-parallel and C-parallel equals twice the C-series the network has two resonant frequencies in the ratio $2: 1$ with $f_{1}=1$ / $(4 \pi \sqrt{L C})$ and $f_{2}=1 /(2 \pi \sqrt{L C})$. The current flowing through the FAIMS device, and hence the voltage across it, is determined by the output impedance of the power amplifier and the ratio of $L$ to $C$. With the circuit values thus chosen, the peak-to-peak voltage across the FAIMS electrodes is $\sim 6 \mathrm{kV}$ when the amplifier outputs $\sim 52$ Watts and $f_{1} \sim 715 \mathrm{kHz}$. This corresponds to a dispersion voltage of $-4 \mathrm{kV}$, and a dispersion field of 20 $\mathrm{kV} / \mathrm{cm}$ for our electrode configuration.

\section{FAIMS Electrodes}

We manufactured cylindrical-domed FAIMS electrodes to sub-micron tolerances from 316 stainless steel following previous designs [16-18]. The inner electrode had a radius of $4 \mathrm{~mm}$, while the outer electrode had a radius of $6 \mathrm{~mm}$. These radii were kept constant at the hemispherical cap of the electrodes, resulting in a gap of 2 $\mathrm{mm}$ throughout the analytical region. See the Supplementary Materials for more details on the design and construction of our FAIMS electrodes.

\section{FAIMS-MS Experiments}

In each of two demonstration experiments, the FAIMS was interfaced with an LTQ ion trap mass spectrometer, manufactured by Thermo Scientific (San Jose, CA, USA).

In the first experiment (Figure 3), we infused a solution of human angiotensin I (Sigma, St. Louis, MO, USA), dissolved in 50:50 methanol:water at $1 \mu \mathrm{M}$ concentration, directly into the FAIMS-MS apparatus at a flow rate of $3 \mu \mathrm{L} / \mathrm{min}$. The outer electrode was biased at $50 \mathrm{~V}$, to match the voltage on the LTQ's heated transfer tube, and the DC bias on the inner electrode was scanned from 30 to $50 \mathrm{~V}$. Therefore, the compensation voltage, defined as the difference in applied field between the inner and outer electrodes, was scanned from -20 to $0 \mathrm{~V}$ (corresponding to a compensation field of -100 to $0 \mathrm{~V} / \mathrm{cm})$. CV was scanned at a rate of 0.1 $\mathrm{V} / \mathrm{s}$, while the mass spectrometer continuously col- 


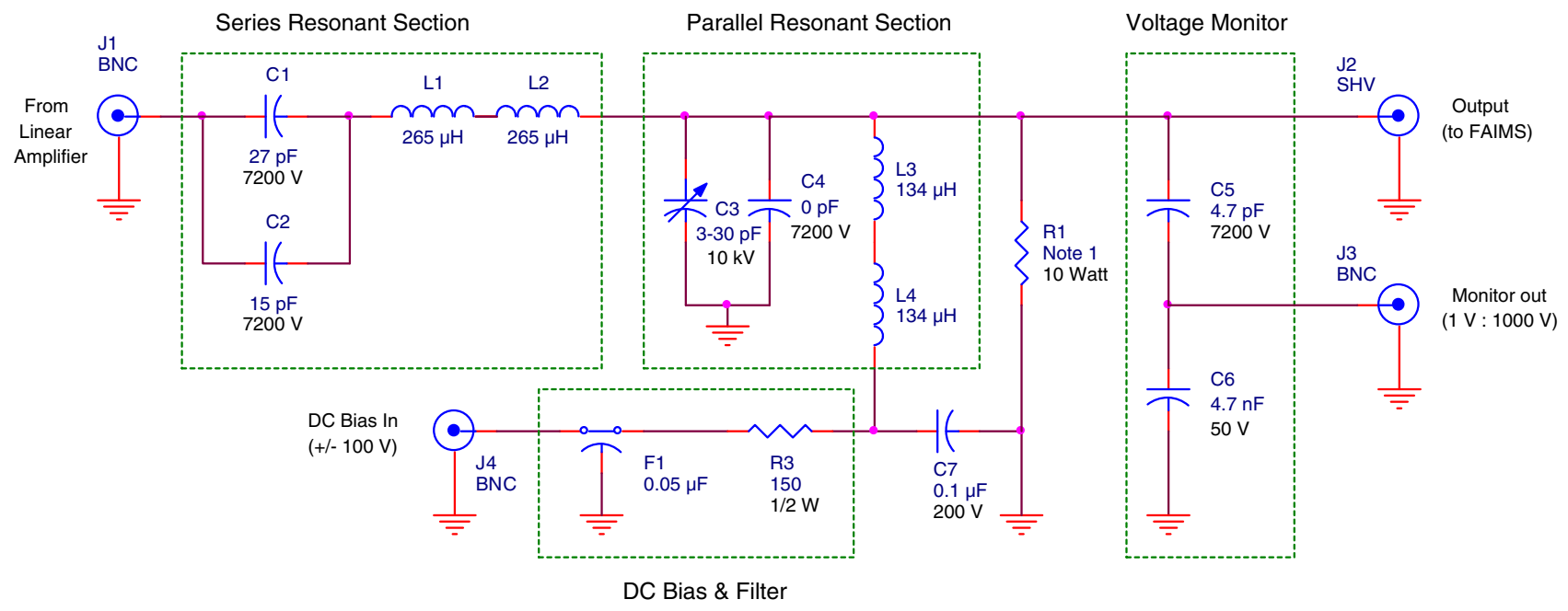

Figure 2. Schematic of the LC coupling network used to supply the dispersion and compensation voltages for FAIMS. (a) rf input is at top left ("from linear amplifier"); compensation voltage input is at bottom left ("DC bias in"), with voltage outputs at right. (b) Capacitor C3 can be any variable capacitor in the needed tuning range, with a minimum voltage rating of $7.5 \mathrm{kV}$. Capacitor $\mathrm{C} 4$ is removed in our circuit, hence the indicated value of $0 \mathrm{pF}$. Additional capacitance may be added to the circuit by raising this value from zero, for the purpose of tuning to specific frequencies.

lected mass spectra over the range $\mathrm{m} / \mathrm{z} 400-1400$. Our carrier gas flow rate was $\sim 3 \mathrm{~L} / \mathrm{min}$ and our curtain plate was biased at $400 \mathrm{~V}$. We compared these data with similar data obtained from the FAIMS apparatus manufactured by Thermo Scientific [19]. In both cases, pure nitrogen was used as the carrier gas. In the Thermo FAIMS device, operational parameters were as follows: dispersion voltage, $-5 \mathrm{kV}$; carrier gas flow rate, $3 \mathrm{~L} / \mathrm{min}$; inner and outer electrode temperature, $70^{\circ} \mathrm{C}$; outer electrode bias, $35 \mathrm{~V}$; entrance plate voltage, $1000 \mathrm{~V}$. CV was scanned using an instrument method that collected one spectrum (with 10 microscans) at each interval of $0.5 \mathrm{~V}$ between -30 and $0 \mathrm{~V}$. The LTQ's front-end optics were tuned separately (using the instrument's autotune routine) for each FAIMS device.

In a second experiment, we performed a simple LC-FAIMS-MS analysis using our hardware. See the Supplementary Materials for details.

\section{Results and Discussion}

To show that our FAIMS device is capable of separating charge states of an individual peptide, we analyzed a solution of human angiotensin I, as described above. Data are shown in Figure 3. The $2+$ and $3+$ charge states of angiotensin are clearly separated, with a resolution that compares favorably with that of Thermo Scientific's FAIMS device. Fitting Gaussian profiles to the peaks in Figure 3a and b, we find that the widths (FWHM) of the fitted Gaussians for the $3+$ charge states are 0.047 and $0.057 \mathrm{Td}$ for our apparatus and Thermo's FAIMS, respectively. For the $2+$ charge state, the widths are 0.034 and $0.047 \mathrm{Td}$ for our apparatus and Thermo's FAIMS, respectively. The shoulders in both datasets are likely due to the appearance of additional conformers not resolved by either device [20].
The difference in observed resolution between the two FAIMS interfaces can be explained by differences in the instrument hardware. The primary differences are (1) a narrower analytical gap in our device of $2 \mathrm{~mm}$ versus $2.5 \mathrm{~mm}$ in the Thermo system, and (2) a slightly longer ion residence time in our system. We calculate, based on gas dynamics considerations, that ions in our device have a residence time of around $130 \mathrm{~ms}$, compared with the $\sim 100 \mathrm{~ms}$ indicated by the default " $\mathrm{CV}$ dwell time" parameter in the Thermo system. This helps explain the observed difference in resolution between the two systems. The two instruments have similar dispersion field strengths due to compensating geometrical and electrical parameters (gap width of 2 $\mathrm{mm}$ and dispersion voltage of $-4 \mathrm{kV}$ for our instrument, versus $2.5 \mathrm{~mm}$ and $-5 \mathrm{kV}$ for Thermo's instrument). The differences in observed compensation voltages are likely due to differences in electrode temperature. The electrodes in the Thermo system were held at $70{ }^{\circ} \mathrm{C}$, whereas the electrodes in our system were subject to heating only from the mass spectrometer's heated capillary. This temperature was measured on the outer electrode to be about $35^{\circ} \mathrm{C}$. Converting to Townsend units, which take into account differences in pressure and temperature across different instruments and laboratories [21], we see that the Thermo system has a dispersion field of about $94 \mathrm{Td}$ whereas our system is operating at $84 \mathrm{Td}$. This difference in dispersion field, combined with a similar correction for the compensation fields, helps explain the difference in observed peak position for the two systems.

\section{Conclusions}

In recent years, FAIMS has become a powerful analytical tool to augment a wide variety of mass spectrometry analyses. Because of the complexity of constructing FAIMS 

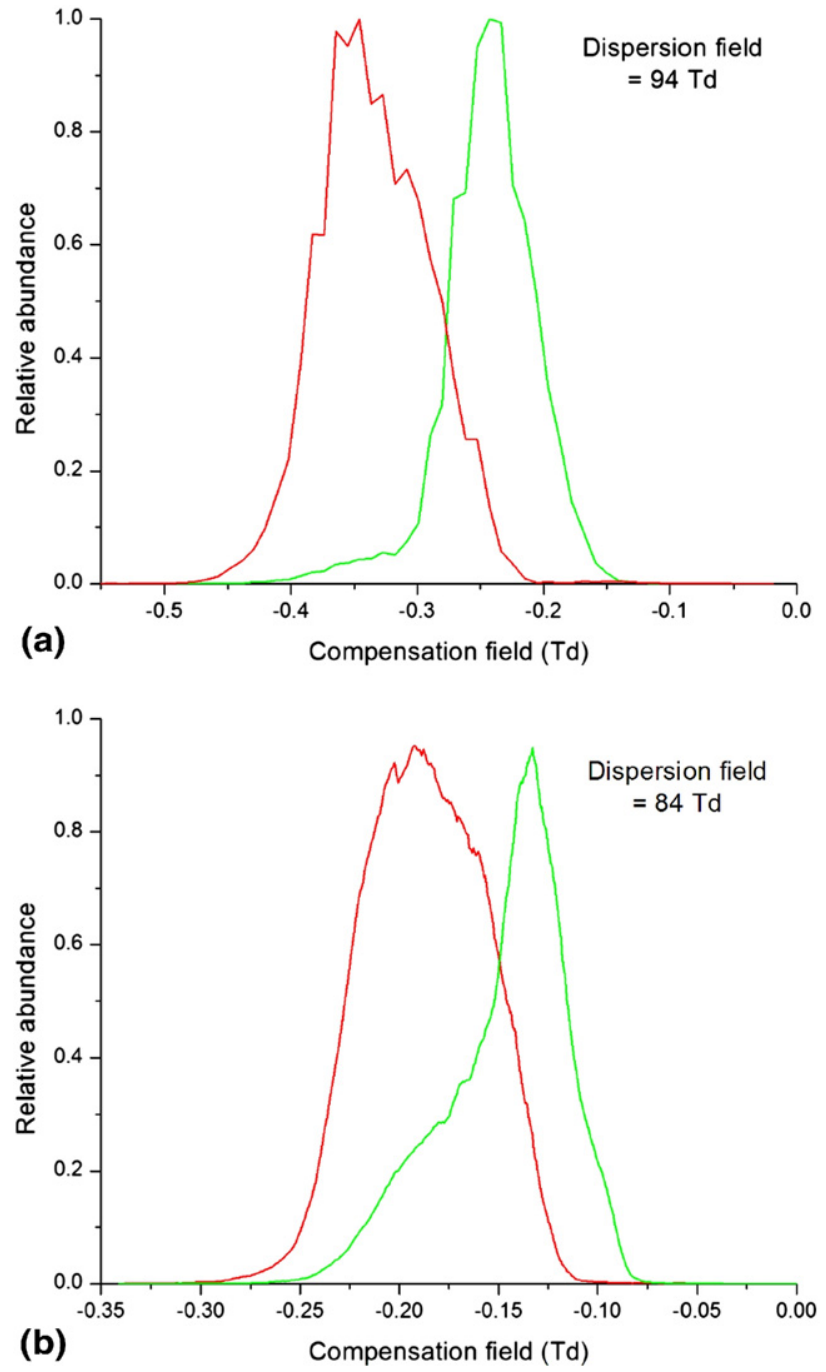

Figure 3. Separation of charge states of angiotensin $\mathrm{I}(+3$, red; +2 , green) in the (a) Thermo FAIMS apparatus and in (b) our FAIMS apparatus. Differences in operational temperature lead to differences in fields expressed in Townsend units; these differences help account for differences in observed peak positions between the two instruments. Peak width and resolution compare favorably.

hardware, especially the driving electronics, only a few labs have constructed their own devices [10-12, 22].

With the goal of producing customizable FAIMS instrumentation, we have constructed a FAIMS system, using traditional cylindrical electrodes with a hemispherical cap in addition to custom-built rf electronics. We have presented data demonstrating the use of this system in FAIMS-MS experiments showing the separation of charge states of angiotensin, as well as in LC-FAIMS-MS experiments showing the separation of peptides in a complex mixture (see the Supplementary Materials).

\section{Acknowledgments}

The authors gratefully acknowledge Larry Stark in the machine shop in the Department of Physics at the University of Washington for his work in manufacturing our FAIMS electrodes. The authors also acknowledge Michael Belford of Thermo Scientific for helpful discussions. Support for this work was provided in part by National Institutes of Health grants P41 RR011823 and an unrestricted Basic Research Grant from Merck Research Laboratories.

\section{Appendix A Supplementary Material}

Supplementary material associated with this article may be found in the online version at doi:10.1016/ j.jasms.2010.02.019.

\section{References}

1. Gorshkov, M. P. Method for Analysis of Additives to Gases, USSR Inventor's Certificate 966,583. 1982.

2. Buryakov, I. A.; Krylov, E. V.; Nazarov, E. G.; Rasulev, U. Kh. A New Method of Separation of Multi-Atomic Ions by Mobility at Atmospheric Pressure Using a High-Frequency Amplitude-Asymmetric Strong Electric Field. Int. J. Mass Spectrom. Ion Processes 1993, 128(3), 143-148.

3. Shvartsburg, A. A. Differential Ion Mobility Spectrometry: Nonlinear Ion Transport and Fundamentals of FAIMS; CRC Press: Boca Raton, 2009;

4. Shvartsburg, A. A.; Tang, K.; Smith, R. D.; Holden, M.; Rush, M.; Thompson, A.; Toutoungi, D. Ultrafast Differential Ion Mobility Spectrometry at Extreme Electric Fields Coupled to Mass Spectrometry. Anal. Chem. 2009, 81(19), 8048-8053.

5. Shvartsburg, A. A.; Smith, R. D.; Wilks, A.; Koehl, A.; Ruiz-Alonso, D.; Boyle, B. Ultrafast Differential Ion Mobility Spectrometry at Extreme Electric Fields in Multichannel Microchips. Anal. Chem. 2009, 81(15), 6489-6495.

6. Kouznetsov, V. High Voltage Waveform Generator; US Patent 5801379 , Sep 1, 1998.

7. McCracken, I. Automated Optimization of Asymmetric Waveform Generator LC Tuning Electronics. US Patent 7058528 B2, Jun 6, 2006.

8. Potvin, L. Baribeau, Y. Waveform Generator Electronics Based on Tuned LC Circuits. US Patent 7078678, Jul 18, 2006.

9. Krylov, E. V. Pulses of Special Shapes Formed on a Capacitive Load. Instrum. Exp. Techniques 1997, 40(5), 628-631.

10. Miller, R. A.; Eiceman, G. A.; Nazarov, E. G.; King, A. T. A Novel Micromachined High-Field Asymmetric Waveform-Ion Mobility Spectrometer. Sensors Actuators B Chem. 2000, 67(3), 300-306.

11. Papanastasiou, D.; Wollnik, H.; Rico, G.; Tadjimukhamedov, F.; Mueller, W.; Eiceman, G. A. Differential Mobility Separation of Ions Using a Rectangular Asymmetric Waveform. J. Phys. Chem. A 2008, 112(16), 3638 3645 .

12. Ridgeway, M. E.; Remes, P. M.; McKinney, C.; Glish, G. L. Radio Frequency Power Supply for the Production of High Amplitude Asymmetric Waveforms. In Proceedings of the 56th ASMS Conference on Mass Spectrometry and Allied Topics; Denver, CO, 2008.

13. Pervukhin, V. V.; Sheven, D. G. Ion Peak Narrowing by Applying Additional AC Voltage (Ripple Voltage) to FAIMS Extractor Electrode. J. Am. Soc. Mass Spectrom. 2010, 21(1), 47-52.

14. Shvartsburg, A. A.; Smith, R. D. Optimum Waveforms for Differential Ion Mobility Spectrometry (FAIMS). I. Am. Soc. Mass Spectrom. 2008 19(9), 1286-1295.

15. Shvartsburg, A. A.; Mashkevich, S. V.; Smith, R. D. Feasibility of Higher-Order Differential Ion Mobility Separations Using New Asymmetric Waveforms. J. Phys. Chem. A 2006, 110(8), 2663-2673.

16. Guevremont, R.; Barnett, D. A.; Purves, R. W.; Vandermey, J. Analysis of a Tryptic Digest of Pig Hemoglobin Using ESI-FAIMS-MS. Anal. Chem. 2000, 72(19), 4577-4584.

17. Guevremont, R.; Purves, R. Comparison of Experimental and Calculated Peak Shapes for Three Cylindrical Geometry FAIMS Prototypes of Differing Electrode Diameters. J. Am. Soc. Mass Spectrom. 2005, 16(3), 349-362.

18. Guevremont, R.; Thekkadath, G.; Hilton, C. K. Compensation Voltage (CV) Peak Shapes Using a Domed FAIMS with the Inner Electrode Translated to Various Longitudinal Positions. J. Am. Soc. Mass Spectrom. 2005, 16(6), 948-956.

19. Barnett, D. A.; Belford, M.; Dunyach, J. J.; Purves, R. W. Characterization of a Temperature-Controlled FAIMS System. J. Am. Soc. Mass Spectrom. 2007, 18(9), 1653-1663.

20. Robinson, E. W.; Sellon, R. E.; Williams, E. R. Peak Deconvolution in High-Field Asymmetric Waveform Ion Mobility Spectrometry (FAIMS) to Characterize Macromolecular Conformations. Int. J. Mass Spectrom. 2007, 259(1/3), 87-95

21. Nazarov, E. G.; Coy, S. L.; Krylov, E. V.; Miller, R. A.; Eiceman, G. A Pressure Effects in Differential Mobility Spectrometry. Anal. Chem. 2006 78(22), 7697-7706.

22. Buryakov, I. A. Express Analysis of Explosives, Chemical Warfare Agents, and Drugs with Multicapillary Column Gas Chromatography and Ion Mobility Increment Spectrometry. J. Chromatogr. B Anal. Technol. Biomed. Life Sci. 2004, 800(1/2), 75-82. 